\title{
Diffusion-based gradient index optics for infrared imaging
}

\author{
Daniel Gibson, ${ }^{\text {a, } * \text { Shyam Bayya, }},{ }^{\mathrm{a}}$ Vinh Nguyen, ${ }^{\mathrm{a}}$ Jason Myers, ${ }^{\text {a Erin Fleet, }}{ }^{\mathrm{a}}$ \\ Jas Sanghera, ${ }^{\mathrm{a}}$ Jay Vizgaitis, ${ }^{\mathrm{b}}$ John Deegan, ${ }^{\mathrm{c}}$ and Guy Beadie ${ }^{\mathrm{d}}$ \\ ${ }^{a}$ US Naval Research Laboratory, Code 5600, Washington, DC, United States \\ ${ }^{\mathrm{b}}$ OptX Imaging, Lorton, Virginia, United States \\ ${ }^{c}$ Rochester Precision Optics, West Henrietta, New York, United States \\ ${ }^{\mathrm{d}}$ Peak Nano, Coppell, Texas, United States
}

\begin{abstract}
New moldable, infrared (IR) transmitting glasses and diffusion-based gradient index (GRIN) optical glasses enable simultaneous imaging across multiple wavebands including short-wave infrared, midwave infrared, and long-wave infrared, and offer potential for both weight savings and increased performance in optical sensors. Lens designs show potential for significant reduction in size and weight and improved performance using these materials in homogeneous and GRIN lens elements in multiband sensors. An IR-GRIN lens with $\Delta n=0.2$ is demonstrated. (C) The Authors. Published by SPIE under a Creative Commons Attribution 4.0 Unported License. Distribution or reproduction of this work in whole or in part requires full attribution of the original publication, including its DOI. [DOI: 10.1117/1.OE.59.11.112604]
\end{abstract}

Keywords: gradient-index lenses; infrared imaging; optical materials; multispectral imaging.

Paper 20200307SS received Mar. 15, 2020; accepted for publication Jun. 2, 2020; published online Jun. 17, 2020.

\section{Introduction}

Recent advances in infrared (IR) detector technology combined with the increasing need within military imaging communities to simultaneously image in multiple IR wavebands have exposed the inadequacy of the currently available IR materials to achieve broadband imaging systems with acceptable system performance compatible with platforms constrained by size, weight, power, and cost (SWaP-C), such as airborne and soldier-borne systems. ${ }^{1}$ While the development of IR focal plane arrays (FPAs) has progressed significantly in recent years, ${ }^{2}$ largely on the coattails of commercial detectors for visible applications, development of materials for IR lenses has lagged behind. When the pixel size of IR FPAs was large ( $>25 \mu \mathrm{m})$, the FPA was the limiting factor in the resolution of the imaging system. The lenses on such systems only needed to resolve about 20-line pairs/mm or less. State-of-the-art FPAs now have pixels as small as $5 \mu \mathrm{m},{ }^{3}$ and the lenses used with them must have higher resolution in order not to degrade the total system performance. The modulation transfer functions (MTFs) of lenses in these systems need to be close to the diffraction limit out to about 70 - to 100 -line pairs $/ \mathrm{mm}$. Meeting this design criteria requires the lens designer to utilize multiple lens elements with large differences in their refractive indexes and dispersions to limit optical aberrations. This is a challenge with the currently limited number of commercially available IR lens materials that transmit broadband IR and have different refractive indexes and dispersions. Chromatic aberration correction over a wide wavelength range is crucial for dual-band or wideband IR systems and benefits from a broad toolbox of materials. Researchers at the U.S. Naval Research Laboratory (NRL) have previously reported new broadband homogeneous chalcogenide glasses to address this issue $\mathrm{e}^{3-5}$ and are in the process of transitioning those materials to industry for manufacturing. Refractive index data for these glasses were published previously ${ }^{6}$ and were available for Zemax OpticStudio and Synopsis CodeV from the authors.

Gradient index (GRIN) materials have carefully tailored optical properties that vary internally within the material in a well-controlled manner. The internal GRIN profile, when combined

*Address all correspondence to Daniel Gibson, E-mail: daniel.gibson@nrl.navy.mil 
with refraction from the surfaces of the optic, provides the optical designer an additional degree of freedom that may be used in various ways including providing additional optical power or correction of chromatic aberration. ${ }^{7}$ GRIN optics for visible wavelengths have been found in nature in the eyes of humans ${ }^{8}$ and fish,, 90 and have been demonstrated in laboratory environments ${ }^{11-13}$ and commercialized in limited sizes and quantities. Various techniques have been utilized to fabricate GRIN optics for visible light including glass element diffusion, ${ }^{14}$ ion exchange in glass, ${ }^{15}$ and polymer nanolayer extrusion. ${ }^{16}$ Recent efforts funded by the DARPA M-GRIN program ${ }^{17}$ have reinvigorated interest in the development of GRIN materials, especially in the $\mathrm{IR}^{3-5,18-20}$ where correction of chromatic aberration is a key design challenge. To date, GRIN optics for IR are not commercially available with the notable exception that some GRIN lenses designed for visible wavelengths also have incidental utility in the nearIR at wavelengths shorter than about $1.5 \mu \mathrm{m}$. The IR-GRIN fabrication technique presented in this paper is based on diffusion of homogeneous chalcogenide glass elements. Other IR-GRIN methods have recently been demonstrated that do not rely on diffusion of glass elements. ${ }^{21}$ Selective ceramization exploits the refractive index change that occurs when specially formulated chalcogenide glasses undergo crystallization..$^{22}$ This can be spatially controlled using laser-induced nucleation ${ }^{23}$ and thermally controlled nucleation and growth ${ }^{24}$ to create GRIN profiles. Electrospray deposition has been shown to produce large index gradients $(\Delta n=0.4)$ in thin films and shows promise for imaging optics. ${ }^{25}$ Ink-jet printing is used commercially at Voxtel Inc. for GRIN optics, primarily in the visible, but can be extended to the IR using suitable inks. ${ }^{26}$ Thermal poling has been used to create GRIN profiles with small gradients $(\Delta n=0.05)$ suitable for microlens arrays. ${ }^{27}$

\section{Moldable Glasses for IR Optics}

Early in the IR-GRIN lens development effort at NRL, a design concept utilizing multiple discrete layers of different IR-transmitting chalcogenide-based glasses was proposed. ${ }^{28}$ In this concept, multilayered lens would have many individual layers that would ultimately be very thin, on the order of the wavelength of light, and approximate a GRIN material through miniaturization. Early designs, however, showed that if the glasses had different dispersions, significant optical benefits could be realized using only a few layers with mm-scale thicknesses and curvature applied to the internal surfaces. By controlling the concentrations and species of chalcogen and metallic elements, a series of IR-transmitting chalcogenide glasses was developed with different refractive indexes and dispersions, but with similar softening temperatures and thermal expansion coefficients. This enables thermal bonding between these glasses and comolding of multilayered lenses. Multilayered lenses were demonstrated in that early work, but proved costly to fabricate and challenging to optimize in design. However, the moldable homogeneous IR-glasses spawned by that work have received continued interest as they can fit into existing design workflows through custom glass catalogs in optical design software such as Zemax OpticStudio and Synopsis CodeV. These chalcogenide glasses are also compatible with conventional manufacturing workflows, including precision glass molding (PGM), grinding, polishing, and single point diamond turning (SPDT). Processing parameters are similar for other commercially available chalcogenide-based glasses.

\subsection{Homogeneous Glass Development for IR Optics}

In this work, 24 IR-transmitting glasses were developed and labeled NRL-1 through NRL-23 and NRL-103. The physical properties of each are summarized in Table 1 . The density of each glass was measured via the Archimedes method with helium gas in a Micromeritics Pycnometer with annealed solid rods $\sim 1 \mathrm{~cm}$ diameter by $1 \mathrm{~cm}$ long. For each glass, the glass transition temperature reported here is the tangent onset temperature of the heat flow curve measured using a TA Instruments Q200 differential scanning calorimeter in hermetically sealed chromated aluminum (Alodine) pans. The coefficients of thermal expansion (CTE) represent the average thermal expansion measured using a pushrod dilatometer. 
Table 1 Physical properties of IR-transmitting chalcogenide glasses developed at NRL.

\begin{tabular}{|c|c|c|c|}
\hline Glass & Density $\left(\mathrm{g} / \mathrm{cm}^{3}\right)$ & $\operatorname{Tg}\left({ }^{\circ} \mathrm{C}\right)$ & CTE $\left(\mathrm{ppm} /{ }^{\circ} \mathrm{C}\right)$ \\
\hline NRL-1 & 3.210 & 195 & 26.6 \\
\hline NRL-2 & 4.554 & 196 & 23.2 \\
\hline NRL-3 & 3.274 & 196 & 25.6 \\
\hline NRL-4 & 4.479 & 201 & 30.2 \\
\hline NRL-5 & 4.421 & 192 & 26.0 \\
\hline NRL-6 & 5.234 & 191 & 18.3 \\
\hline NRL-7 & 3.177 & 201 & 25.3 \\
\hline NRL-8 & 4.516 & 193 & 25.7 \\
\hline NRL-9 & 3.281 & 197 & 25.1 \\
\hline NRL-10 & 4.446 & 191 & 27.0 \\
\hline NRL-11 & 3.278 & 196 & 25.5 \\
\hline NRL-12 & 3.403 & 193 & 25.9 \\
\hline NRL-13 & 3.539 & 192 & 25.2 \\
\hline NRL-14 & 3.660 & 184 & 23.3 \\
\hline NRL-15 & 3.776 & 183 & 24.7 \\
\hline NRL-16 & 3.897 & 182 & 24.0 \\
\hline NRL-17 & 4.026 & 181 & 22.7 \\
\hline NRL-18 & 4.134 & 180 & 22.8 \\
\hline NRL-19 & 4.252 & 178 & 22.4 \\
\hline NRL-20 & 4.365 & 183 & 23.5 \\
\hline NRL-21 & 4.480 & 180 & 22.5 \\
\hline NRL-22 & 4.587 & 178 & 22.9 \\
\hline NRL-23 & 4.584 & 177 & 22.6 \\
\hline NRL-103 & 4.540 & 314 & 14.0 \\
\hline
\end{tabular}

Optical properties are reported in Table 2 and include the IR transmission window, refractive index, thermo-optic coefficient $(d n / d T)$, and dispersion for each of the three IR wavebands, short-wave (SWIR, 1 to $3 \mu \mathrm{m}$ ), midwave (MWIR, 3 to $5 \mu \mathrm{m}$ ), and long-wave infrared (LWIR, 8 to $12 \mu \mathrm{m}$ ). The IR transmission window is presented here as the short and long wavelength cut-off edges where the transmission drops to $50 \%$ of the maximum. The spectral transmission for each glass was measured using polished flat thin samples (typically $2.0 \mathrm{~mm}$ thick) with parallel faces on an Analect FTIR. The refractive index for each glass was measured by $\mathrm{M}^{3} \mathrm{MSI}$ (Escondido, California) using minimum deviation refractometry on both wedge samples (for room temperature measurements) and prism samples (for temperature-dependent measurements between $250 \mathrm{~K}$ and $350 \mathrm{~K}$ ). The refractive index data are summarized here, but complete data are available in the form of a datafile compatible with Zemax OpticStudio and Synopsis CodeV from the authors. Dispersion fit and thermal fit parameters were presented previously ${ }^{6}$ as well as a comprehensive overview of the fitting method. All glasses in this series transmit SWIR, MWIR, and LWIR with the exception of NRL-6, which has only partial SWIR coverage down to about $1.4 \mu \mathrm{m}$. 
Table 2 Optical properties of IR-transmitting glasses developed at NRL. -3-dB window is defined as the short and long wavelengths, in $\mu \mathrm{m}$, where the transmission, measured using FTIR on 2-mm-thick polished samples, drops to $50 \%$ of the maximum. For each waveband, $n$ and $d n / d T$ in $p p m /{ }^{\circ} \mathrm{C}$ are reported for the center wavelength of the band.

\begin{tabular}{|c|c|c|c|c|c|c|c|c|c|c|c|}
\hline \multirow[b]{2}{*}{ Glass } & \multicolumn{2}{|c|}{$\begin{array}{c}-3-\mathrm{dB} \\
\text { window }\end{array}$} & \multicolumn{3}{|c|}{ SWIR ( 1 to $3 \mu \mathrm{m})$} & \multicolumn{3}{|c|}{ MWIR ( 3 to $5 \mu \mathrm{m}$ ) } & \multicolumn{3}{|c|}{ LWIR (8 to $12 \mu \mathrm{m}$ ) } \\
\hline & $\lambda_{\text {short }}$ & $\lambda_{\text {long }}$ & $n_{2 \mu \mathrm{m}}$ & $d n / d T$ & V & $n_{4 \mu \mathrm{m}}$ & $d n / d T$ & $V$ & $n_{10 \mu \mathrm{m}}$ & $d n / d T$ & $V$ \\
\hline NRL-1 & 0.58 & 12.7 & 2.41383 & -15.7 & 24 & 2.39919 & -17.8 & 150 & 2.37044 & -17.9 & 49 \\
\hline NRL-2 & 0.79 & 18.2 & 2.72921 & 43.8 & 15 & 2.70681 & 37.4 & 159 & 2.69009 & 36.1 & 142 \\
\hline NRL-3 & 0.89 & 12.5 & 2.41417 & -3.9 & 23 & 2.39893 & -6 & 148 & 2.37042 & -5.78 & 49 \\
\hline NRL-4 & 0.78 & 17.5 & 2.66815 & -17.3 & 17 & 2.64837 & -18.6 & 186 & 2.63709 & -18.3 & 233 \\
\hline NRL-5 & 0.74 & 17.2 & 2.49378 & 9.61 & 19 & 2.47761 & 7.62 & 184 & 2.46289 & 6.33 & 126 \\
\hline NRL-6 & 1.43 & 18.2 & 3.22366 & 202 & & 3.17169 & 169 & 107 & 3.15227 & 160 & 257 \\
\hline NRL-7 & 0.57 & 12.7 & 2.40106 & -4.38 & 24 & 2.38657 & -7.34 & 159 & 2.3576 & -8.11 & 47 \\
\hline NRL-8 & 0.79 & 18.9 & 2.68413 & 2.97 & 16 & 2.66324 & 1.2 & 184 & 2.64996 & -0.40 & 186 \\
\hline NRL-9 & 0.61 & 12.8 & 2.43256 & -3.64 & 23 & 2.41739 & -3.83 & 160 & 2.38929 & -5.94 & 51 \\
\hline NRL-10 & 0.74 & 17.5 & 2.54364 & 27.2 & 18 & 2.52625 & 22.6 & 191 & 2.51154 & 21 & 135 \\
\hline NRL-11 & 0.62 & 12.9 & 2.44475 & -0.26 & 23 & 2.42945 & -1.56 & 161 & 2.40174 & -4.56 & 52 \\
\hline NRL-12 & 0.64 & 13.0 & 2.47421 & 1.3 & 22 & 2.45826 & 0.59 & 163 & 2.43164 & 0.50 & 57 \\
\hline NRL-13 & 0.66 & 13.0 & 2.50665 & 3.9 & 21 & 2.49008 & 1.2 & 164 & 2.46436 & -0.51 & 61 \\
\hline NRL-14 & 0.71 & 12.7 & 2.53678 & 12 & 20 & 2.51941 & 7.6 & 154 & 2.49471 & 5.6 & 66 \\
\hline NRL-15 & 0.70 & 13.1 & 2.56721 & 19 & 19 & 2.54917 & 14 & 157 & 2.52575 & 11 & 72 \\
\hline NRL-16 & 0.74 & 13.0 & 2.59823 & 21 & 18 & 2.57946 & 14 & 157 & 2.55692 & 12 & 78 \\
\hline NRL-17 & 0.75 & 13.1 & 2.63285 & 25 & 17 & 2.61315 & 19 & 159 & 2.59157 & 17 & 84 \\
\hline NRL-18 & 0.77 & 13.2 & 2.66467 & 29 & 17 & 2.64413 & 22 & 161 & 2.62368 & 20 & 94 \\
\hline NRL-19 & 0.78 & 13.2 & 2.70128 & 27 & 16 & 2.67987 & 20 & 162 & 2.66038 & 17 & 104 \\
\hline NRL-20 & 0.80 & 13.4 & 2.73011 & 20 & 16 & 2.70774 & 16 & 157 & 2.68936 & 13 & 119 \\
\hline NRL-21 & 0.81 & 13.8 & 2.76762 & 45 & 15 & 2.7442 & 36 & 160 & 2.72645 & 33 & 132 \\
\hline NRL-22 & 1.10 & 15.0 & 2.80257 & 48 & 14 & 2.77794 & 39 & 154 & 2.76135 & 36 & 157 \\
\hline NRL-23 & 1.30 & 18.2 & 2.80595 & 32 & 14 & 2.78114 & 25 & 155 & 2.76493 & 22 & 160 \\
\hline NRL-103 & & & 2.73697 & 65 & 16 & 2.71527 & 61 & 170 & 2.70057 & 58 & 167 \\
\hline
\end{tabular}

Windows have been fabricated from all of the materials above using grinding and polishing, and lenses were demonstrated for several of the glasses using SPDT and PGM, several examples of which are shown in Fig. 1. All examples shown here are aspheric lenses with clear apertures of about $25 \mathrm{~mm}$. The SPDT was performed by OptiCraft, Inc. (Woburn, Massachusetts), and PGM lenses were fabricated by Prof. Allen Yi at Ohio State University (Columbus, Ohio). Optical flats and windows were fabricated by Sydor Optics (Rochester, New York) and J.R. Cumberland, Inc. (Marlow Heights, Maryland). Additional lenses have been fabricated by Rochester Precision Optics (West Henrietta, New York) in diameters up to $50 \mathrm{~mm}$. The glasses in this work have been routinely fabricated in 25 - and $55-\mathrm{mm}$ diameter ingots that may be cut into optical blanks. Some glasses have been demonstrated with larger diameters through contracts with commercial 


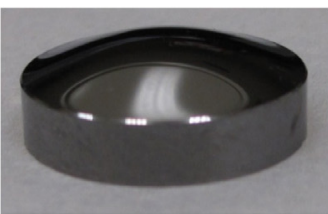

(a)

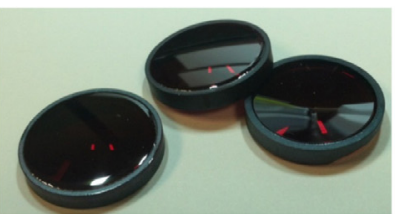

(b)

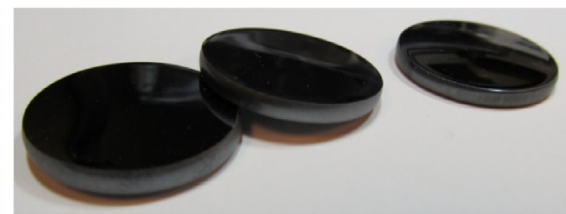

(c)

Fig. 1 Sample lenses using NRL IR-glasses. (a) Aspheric NRL-6 via SPDT, (b) PGM NRL-12, and (c) PGM NRL-8.

glass producers in 100-mm-diameter ingots and 200-mm-diameter plates. While the glasses are compatible with common industrial chalcogenide glass production methods, the materials are not yet commercially available.

\subsection{PseudoGRIN Lens Demonstration}

Many of the glasses developed in this work were designed to have very similar thermophysical properties including thermal expansion coefficient and glass transition temperature $(T g)$ as a surrogate for viscosity behavior. Part of the motivation for that criterion was driven by the desire to thermally bond the glasses to each other to enable bonded achromatic doublets. Such optics are common in systems that operate at visible wavelengths, but are practically nonexistent in the IR. This is due in part to the often extreme mismatch in thermal expansion behavior for common IR materials and a lack of commercially available IR transparent adhesive in spite of historic ${ }^{29-31}$ and more recent ${ }^{32,33}$ work in this area.

In an attempt to demonstrate thermal bonding with these materials, a two-element imaging lens having a 22-mm focal length, $f / \#=1.5$, and a clear aperture of $20 \mathrm{~mm}$ was designed where the front optic comprises four layers of NRL glass bonded together without adhesive. ${ }^{34}$ The optical design, shown in Fig. 2(a), is termed "pseudoGRIN" as it contains a discontinuous refractive index distribution. This unique front element was fabricated by thermally bonding disks of NRL-10, NRL-8, NRL-2, and NRL-6 to each other without any adhesives to form a cylindrical bonded blank. The blank was then molded on a prototype lens molding machine using a concave/convex die set with spherical curvature in order to impart curvature to the three internal conic optical surfaces. Of the 10 blanks that were molded, 4 were sectioned using a low-speed diamond saw and the internal interfaces were mapped using optical microscopy. In order to account for minor deviations from the intended optical design, the measured curvature of the internal interfaces was fed back to the optical design, and the external surfaces of both the multilayered front element and the homogeneous rear element were reoptimized. The aspheric front and rear optical surfaces were formed by SPDT, as were the aspheres on the second, NRL-6, element. The lens was paired with an LWIR thermal imaging camera, FLIR systems Tau2, for demonstration purposes. Test subjects included an adult man and a United States Air Force (USAF) 1951 3-bar template placed in front of an extended source blackbody [Figs. 2(b) and 2(c), respectively]. This is the first successful demonstration of IR imaging using a lens of this type, and it should be noted that none of the lens elements were outfitted with antireflective (AR) coatings.

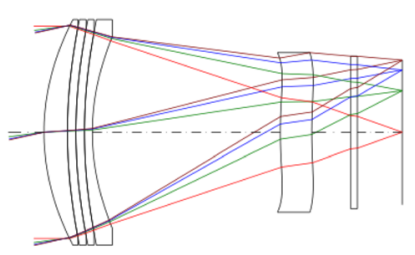

(a)

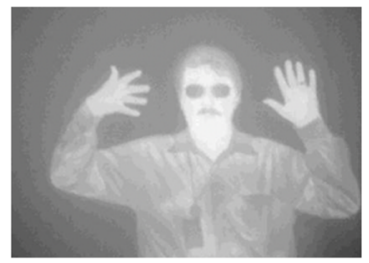

(b)

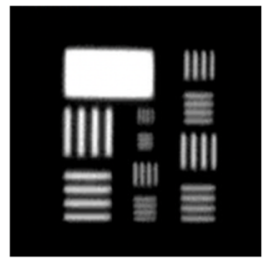

(c)

Fig. 2 (a) Ray tracing schematic of multilayered imager lens design where the front lens element comprises four different IR optical glasses. Sample images from (b) the lens demonstrator include an adult man and (c) a USAF bar chart mask in front of a blackbody source. 
Although the "pseudoGRIN" lens design demonstrated here is of limited practical utility, its demonstration serves to validate the homogeneous IR glasses for use in more conventional optics and paves the way for development of true diffusion-based GRIN using these materials. For example, the pseudoGRIN lens validates the capability to thermally bond these new IR glasses without the need for specialty adhesives. The bonded glass body is sufficiently durable to be successfully comolded using conventional PGM tooling. In addition, bonded and molded glasses can be resurfaced using conventional SPDT machining without delamination or fracture, even with internal layers about 1.0 -mm thick.

Further validation was realized recently by an engineering team at Rochester Precision Optics (RPO) who, in 2018, designed ${ }^{35}$ and subsequently demonstrated in $2019^{29,30}$ a dual-band MWIR-LWIR $3 \times$ zoom lens using homogeneous IR glass materials supplied by NRL. Their design criteria were a $3 \times$ continuous optical zoom lens with a 50 - to $150-\mathrm{mm}$ focal length range and $f / 3$ operating from 3.5 to $11.5 \mu \mathrm{m}$. Jamie Ramsey et al. showed that a lens made entirely from commercially available optical materials (IRG-22, 23, 24, 27, germanium, GaAs, and ZnS) would require 21 lens elements and have a total optical mass of $416 \mathrm{~g}$ for the "baseline design." By incorporating the new NRL glasses into the "SWaP design" in both singlet and bonded doublet forms, the lens element count was reduced to 12 and the total optical mass was halved. The materials used were IRG-24, 27, RPO classic 2, NRL-4, 6, and 8, and ZnS. Notably, germanium and GaAs were eliminated from the system leaving $\mathrm{ZnS}$ as the only crystal. Since the lens element count was drastically reduced, the number of surfaces requiring AR coating was cut in half, potentially reducing costs and increasing optical transmission.

\section{IR-GRIN Fabrication}

The internal refractive index distribution in a GRIN lens, or GRIN profile, could be generalized as a function of position in cylindrical coordinates, and in principle, may take any arbitrary form. The GRIN profiles used in the design of optical systems, however, are typically limited to rotationally symmetric distributions. GRIN fabrication methods can impose further limitations on the GRIN profile design, as is the case in this work. As a result, three types of GRIN profiles are considered here: axial, radial, and hybrid or spherical and are shown schematically in Fig. 3. Axial GRIN has a refractive index profile that changes only in the $z$ direction (coincident with the optical axis) and is orthogonally invariant (in $x$ and $y$ directions). The refractive index profile of a typical radial GRIN element is rotationally symmetric about the optical axis and varies with distance from that axis, $r$, while remaining invariant in $\theta$, and along the optical axis, $z$ (in cylindrical coordinates). A hybrid GRIN has components of both axial and radial GRIN, while maintaining rotational symmetry about the optical axis. One example of this is a spherical gradient, wherein the gradient is a function of $r$ in spherical coordinates and is invariant in $\varphi$ and $\theta$.

The GRIN fabrication method of this work relies on the assembly and subsequent diffusion of homogeneous glass bodies. The resultant diffusion profiles depend on the ability to fabricate and assemble the individual components (glass plates in this work). Extending this method to pure radial GRIN profiles requires the assembly and subsequent diffusion of nested glass tubes and is an area of ongoing work.

\subsection{Diffusion-Based Process}

The IR-GRIN fabrication technique developed at NRL is based on diffusion of chalcogenide glasses ${ }^{18}$ and is schematically shown in Fig. 4. First, separate homogeneous glass elements with

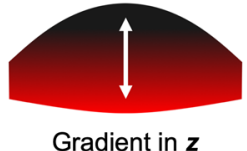

(a)

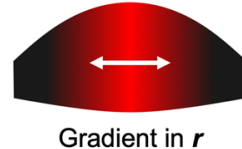

(b)

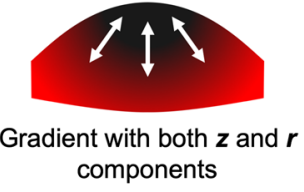

(c)

Fig. 3 GRIN lens profiles can be described as (a) axial, (b) radial, and (c) hybrid. 


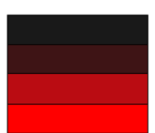

(a)

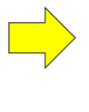

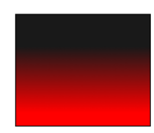

(b)

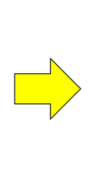

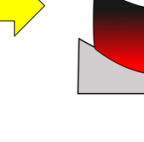

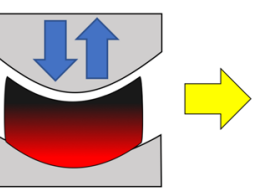

(c)

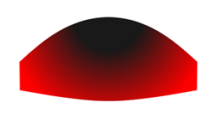

(d)

Fig. 4 (a) The process for fabrication of IR-GRIN optical elements starts with the fabrication of a layered preform comprising several homogeneous glass elements with different elemental compositions. (b) The preform is heated to a prescribed temperature for a prescribed time to allow the glass elements to interdiffuse forming a GRIN blank with a continuously graded internal refractive index profile. (c) Molding is used to deform the axial GRIN blank into a spherical one. (d) The spherical GRIN blank can then be made into a GRIN lens through conventional means.

different elemental compositions are combined in a prescribed orientation in a cleanroom environment. The elements, in this case thin glass plates, are then bonded together at an elevated temperature without any adhesives or voids at the interfaces to produce a preform with discrete layers. The preform is then heated to a prescribed temperature and time schedule to allow the homogeneous glasses to interdiffuse across their interfaces. The resulting GRIN blank has a continuously graded composition and refractive index profile in the direction of the optical axis. This axial GRIN blank can then be molded into a hybrid-spherical GRIN blank using compression molding or slumping between hemispherical molds, which imparts curvature not only to the outer surfaces but also to the internal iso-indicial planes. A hybrid or spherical GRIN profile has components of both axial and radial GRIN, and therefore, can provide optical power. SPDT is then used to generate refractive external surfaces on the axial or hybrid-spherical IR-GRIN blank to form a lens element.

The IR-GRIN process employs homogeneous chalcogenide glasses as their optical and physical properties can be accurately controlled by tailoring their compositions, and the glasses used are a subset of those developed in Sec. 2. Specifically, the homogeneous multicomponent chalcogenide glasses used in the fabrication of IR-GRIN and NRL-11 through NRL-22, are a continuous blend of the NRL-1 and NRL-23 endpoint glasses, making the full $\Delta n$ available for use in IR-GRIN profiles $\sim 0.4$ as shown in the dispersion curves in Fig. 5. The glasses are well-behaved in that there exists a smooth transition in the optical and physical properties through the compositional space. In other words, the glass properties in general (viscosity, glass transition temperature, IR transmission window, density refractive index, and dispersion) vary smoothly and predictably with composition and can be evaluated directly from the compositional gradient as presented elsewhere. ${ }^{36,37}$

\subsection{Diffusion Modeling}

Diffusion behavior in the chalcogenide glasses used here follows Fick's laws of diffusion

$$
\begin{gathered}
J=-D \frac{\partial \phi}{\partial x} \\
\frac{\partial \phi}{\partial t}=D \frac{\partial^{2} \phi}{\partial x^{2}}
\end{gathered}
$$

where $J$ is the diffusion flux, $D$ is the diffusivity, $\phi$ is the concentration, $x$ is the diffusion dimension, and $t$ is the time. In addition, the diffusivity itself is both thermally activated and concentration dependent and thus follows a modified Arrhenius law

$$
D(\phi, T)=D_{0}(\phi) \exp \left(-\frac{Q_{d}}{R T}\right)
$$

where $T$ is the temperature, $Q_{d}$ is the activation energy for diffusion, $D_{0}(\phi)$ is the concentration dependent diffusivity, and $R$ is the ideal gas constant. The diffusion constants were determined using diffusion couplets. 


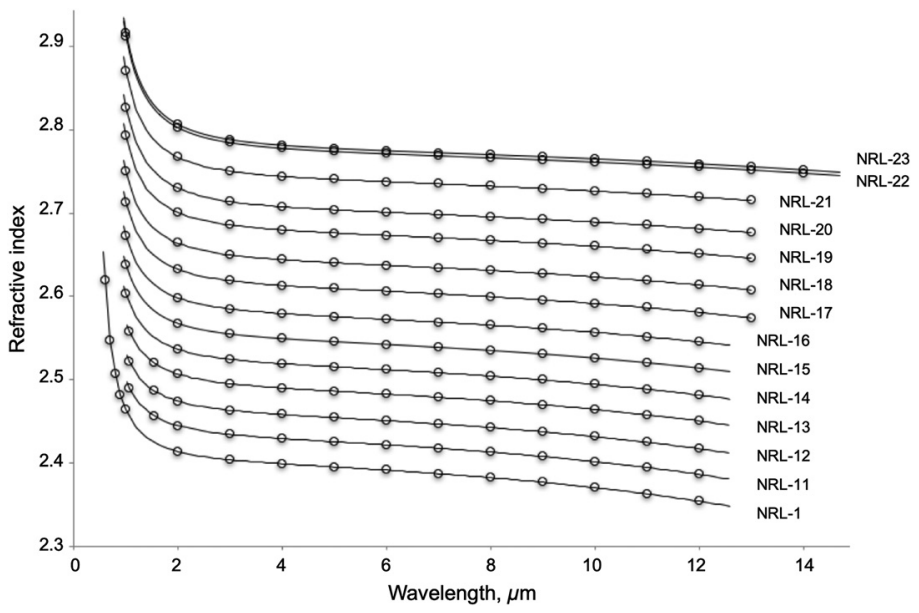

Fig. 5 Refractive index and dispersion were measured for the homogeneous glasses developed for IR-GRIN and include NRL-1, and NRL-11 through NRL-23. The maximum $\Delta n$ available is 0.4.

Diffusion couplets were prepared by first polishing and then thermally bonding pairs of 2.0-mm-thick glass plates comprising each of the endpoint glasses, NRL-1 and NRL-23. The couplets were then heated in a controlled environment for a series of times and different temperatures. The resulting diffused couplets were sectioned and polished, and the compositional gradient was analyzed using electron probe microanalysis (EPMA). The concentration profiles of the individual atomic constituents were mapped using wavelength-dispersive spectroscopy linescan with steps between 1 and $15 \mu \mathrm{m}$ depending on the amount of diffusion observed, an example of which is shown in Fig. 6. The EPMA linescan measurement is a destructive technique and was not performed on these specific samples prior to the diffusion step. During process development, EPMA was used on undiffused samples to confirm that the thermal bonding step, which takes place at a lower temperature and does not introduce any diffusion within the spatial capabilities of the instrument $(\sim 1 \mu \mathrm{m})$. Since the multicomponent glasses in the IR-GRIN process were carefully designed to be a blend of the two endpoint glass compositions, only the relative concentration of either NRL-1 or NRL-23 glass, rather than the concentrations of the individual elements, needs be considered. In the example, the position of the original interface is indicated by the dashed vertical line at a distance $=0 \mu \mathrm{m}$ and the red and green dots indicate the relative concentration of NRL-1 glass and NRL-23 glass, respectively, after diffusion. The diffused region of the couplet in this example is almost $1400-\mu \mathrm{m}$ wide, and the asymmetry in the curves is evidence that diffusion is faster where the concentration of NRL-23 is higher and that the diffusivity is concentration dependent. Although the data presented in Fig. 6 were obtained using the NRL-1 and NRL-23 endpoint glasses, the diffusion

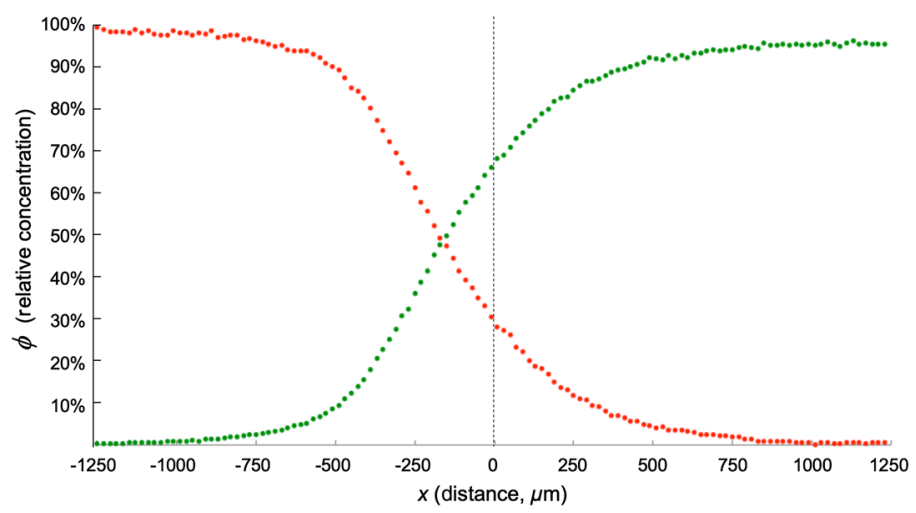

Fig. 6 EPMA was used to profile the interdiffusion of NRL-1 (red dots) and NRL-23 (green dots) glass plates heated for five days in this example. The dashed line at distance $=0$ indicates the position of the interface between the two glasses prior to diffusion. 


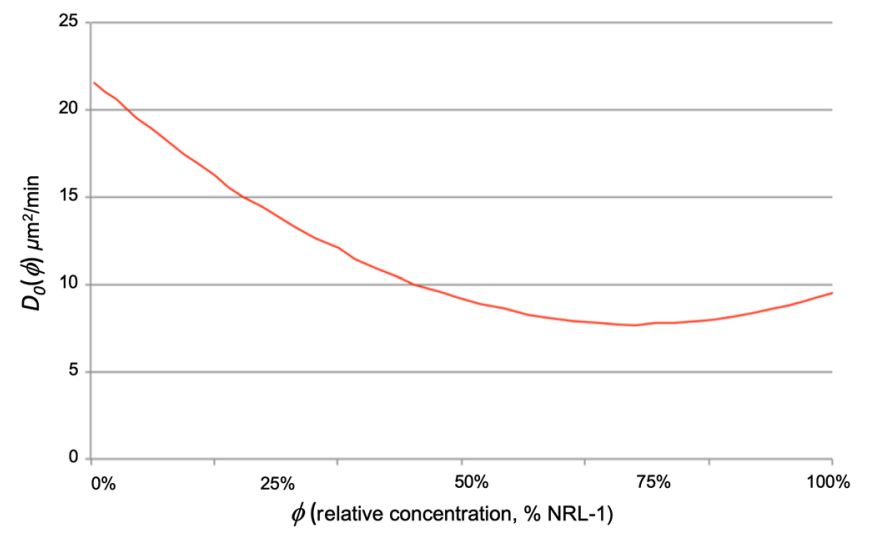

Fig. 7 Concentration-dependent diffusion coefficient, $D_{0}(\phi)$, in the NRL-1/NRL-23 GRIN glass system.

process creates a continuous concentration gradient, which, in effect, creates regions within the sample having compositions identical to the glasses NRL-11 through NRL-22 as well as everything in between. These diffusion couplets thus capture the diffusion information for all of the glass compositions. The concentration-dependent diffusivity, $D_{0}(\phi)$, was calculated from the diffusion profile using Eq. (2) and the Boltzmann-Matano method ${ }^{13}$ and is shown in Fig. 7. In this glass system, the diffusivity was found to vary by a factor of $>2$ within the compositional range of the system.

In order to simulate axial GRIN diffusion in an arbitrary layered preform, a one-dimensional finite volume method was employed. The preform is divided into many small segments in the direction of the gradient with lengths on the order of $<10 \mu \mathrm{m}$. The concentration-dependent diffusivity, $D_{0}(\phi)$, is used to calculate the flux of NRL-1 and NRL-23 at the boundary of each segment according to Fick's first law [Eq. (1)] as well as the new concentration of each segment, as shown schematically in Fig. 8. This is then repeated for many small time increments, $\Delta t$, such that the corresponding diffusion distance, $2(D t)^{1 / 2}$, is less than the length of the segment, $\Delta x$, satisfying the Courant-Friedrichs-Lewy condition. ${ }^{38}$ The simulation was validated against the diffusion couplets, such as the one shown in Fig. 6, and other partially diffused stacks of glass plates including intermediate compositions (e.g., NRL-11, 12, and 13).

This simulation forms the basis for a prescriptive diffusion model. This model was developed to prescribe how a GRIN preform stack should be constructed in order to achieve a desired profile in an axial GRIN preform upon diffusion. To accomplish this, an optimization routine was employed that iterates the diffusion simulation varying both the thicknesses and concentrations of the internal layers and calculates the net deviation from a target concentration profile for each iteration. It is important to note that the diffusion simulation and optimization operate in

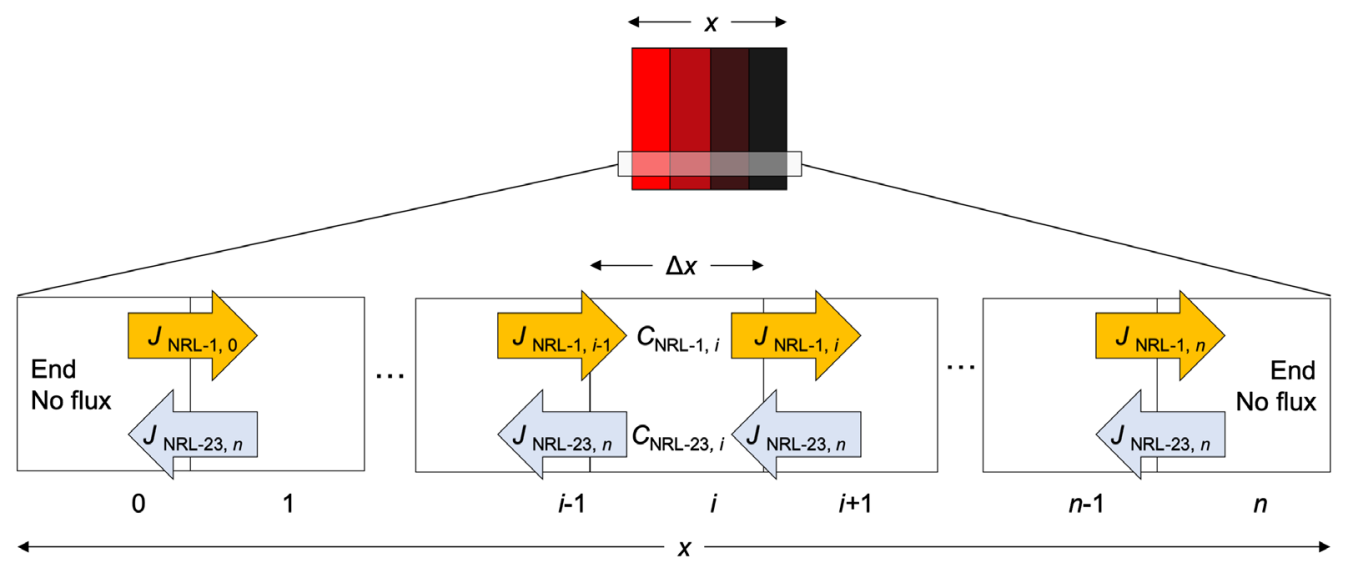

Fig. 8 Schematic for simulating diffusion in one-dimension using a finite volume technique. 
the realm of concentration and not refractive index. Therefore, the GRIN profile designer must consider the dispersion of the material when specifying the target GRIN profile and selecting the endpoint glasses from within the glass series. The optimizer generates a first-guess preform stack by dividing the target concentration profile, a map of $\phi(x)$, into $\sim 1$-mm-thick plates and averaging the concentration over each plate. Diffusion is then simulated over the entire stack and a deviation profile, the difference between the diffusion profile and the target profile, is calculated. The magnitude of the deviation profile is used as a scoring metric to optimize the stack. It is intuitive that the concentration profile can be made smooth by diffusing for a very long time. However, this tends to homogenize the profile. An optimum diffusion time for the stack is determined when the magnitude of the deviation profile starts to increase as fidelity is lost. The optimization routine iterates this process optimizing both the thickness and composition of the glass plates until the deviations are below the specified target.

Deviations of \pm 0.001 refractive index units are typical targets, but this can be reduced by increasing the number of layers and reducing their thicknesses. The prescriptive diffusion model has been adapted to radial profiles but validation is reserved for future work. Hybrid GRIN blanks with spherical internal curvature are not considered in the diffusion model as they are generated by slumping or molding an axial GRIN blank after the diffusion step.

\section{IR-GRIN Demonstration Optic}

A dual-band (MWIR-LWIR) IR imaging lens was designed to demonstrate the capabilities of an IR-GRIN lens element. The lens was designed with a 66 -mm focal length, $f / 2.4$ with a \pm 8 - deg field of view (FOV), and diffraction limited performance over a wavelength range from 3 to $12 \mu \mathrm{m}$. The two-element lens design, shown in Fig. 9, has a thermally bonded IR glass doublet front element and an IR-GRIN rear element with a hybrid-spherical profile. The IR-GRIN lens was specified with a linear $\Delta n$ of about 0.2 , and the diffusion optimization model determined a prescription having five starting layers each with a unique glass composition as shown in Fig. 10. The specified diffusion time was about five days. Two batches of six diffused IR-GRIN blanks were fabricated according to the prescription shown in Table 3, and one sample was randomly selected from each batch for destructive analysis using EPMA. The remaining four blanks were compression molded between spherical molds to hybridize the linear GRIN gradient into a spherical one. The outer surfaces were then generated by SPDT forming the IR-GRIN lens elements, two of which are shown in Fig. 11(a).

One of the lenses was cut for analysis, and a slice 0.8 -mm thick was cut from the center and polished on both sides. The sample was analyzed in a Mach-Zhender interferometer ${ }^{13}$ at $1 \mu \mathrm{m}$ by the GRIN optics lab headed by Prof. Duncan Moore at The University of Rochester (Rochester, New York). The resulting interferogram, shown in Fig. 11(b), reveals a multitude of interference fringes, confirming the existence of a refractive index gradient that was indeed hybridized by the compression molding process and is symmetric about the optical axis as intended. It is apparent from the interferogram that there is a small region near the center with

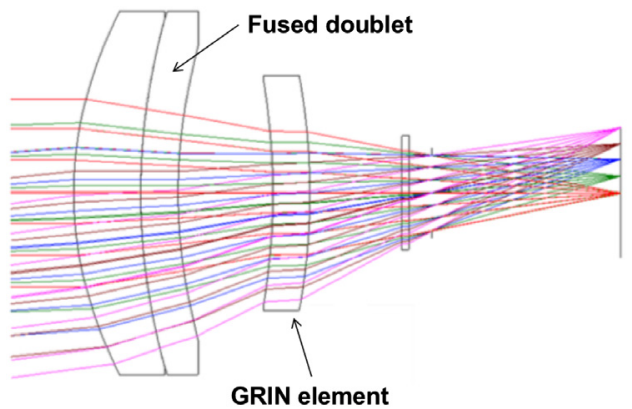

(a)

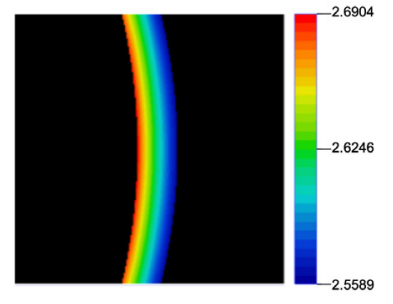

(b)

Fig. 9 (a) IR-GRIN demonstrator design has a front element comprising a fused IR-glass doublet and a GRIN rear element. (b) The rear GRIN element has a hybrid-spherical profile varying linearly with $r$. 


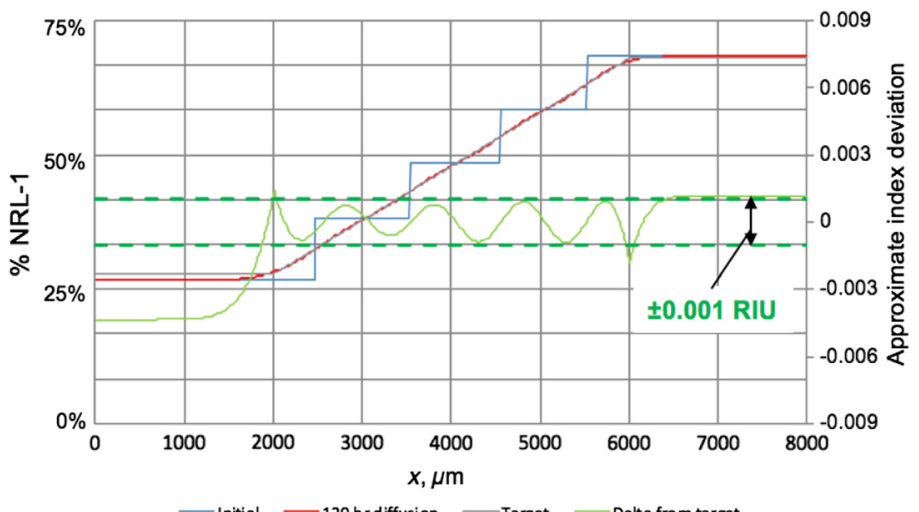

Fig. 10 IR-GRIN prescription for a linear profile, $\Delta n=0.2$, and a specified maximum deviation from target profile of $\pm 0.001 \mathrm{RIU}$. The starting stack prescription is shown in blue, the diffused profile after $120 \mathrm{~h}$ is red, and the predicted deviation is in green (uses right and scale).

Table 3 Starting stack prescription profile before diffusion into IR-GRIN.

\begin{tabular}{lccc}
\hline \hline Layer & Thickness $(\mu \mathrm{m})$ & Composition $(\%$ NRL-1) & Glass ID \\
\hline 1 & 2465 & 26.2 & NRL-19 \\
2 & 1067 & 37.5 & - \\
3 & 1012 & 47.7 & - \\
4 & 984 & 57.4 & - \\
5 & 2472 & 67.2 & NRL-14 \\
\hline \hline
\end{tabular}

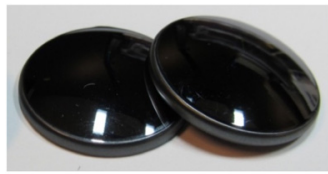

(a)

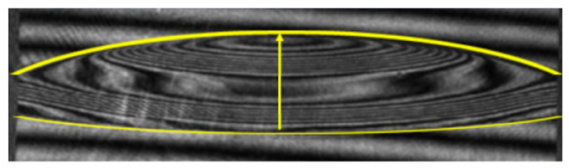

(b)

Fig. 11 (a) GRIN lens elements were fabricated and (b) interferometry revealed a hybrid spherical index gradient with a defect near the center.

low fringe density indicating almost no gradient. Further analysis of undiffused samples confirmed that this was due to a stacking fault in the original preform, where one of the glasses was duplicated in the stack. This was verified using the diffusion simulation with the defective preform configuration.

Unfortunately, the bonded doublet front element was not available to test the imager in its intended configuration and validation of its chromatic performance across the dual-band wavelength range of operation is reserved for future work. A simulation in Zemax revealed that the lens element could function reasonably well as a singlet in the LWIR, 8 to $12 \mu \mathrm{m}$, although it was not optimized for this application. The IR-GRIN lens was inserted into a commercial LWIR camera, specifically a Sig-Sauer thermal reflex sight, and took the place of the dual-element lens in that system. In spite of the defects, the IR-GRIN lens did successfully image a car as shown in Fig. 12. Note that the lens in this configuration was not designed for this system, and it also was not AR-coated contributing to the degraded performance. The IR-GRIN singlet was also evaluated against a USAF 1951 3-bar test pattern line mask in front of a black body source using an FLIR Tau 2 camera core as shown in Fig. 13(a). The lens was able to resolve the test bars adequately, Fig. 13(b), in spite of the defects. 


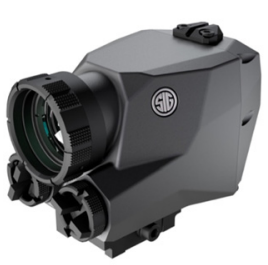

(a)

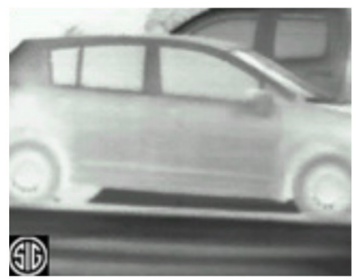

(b)

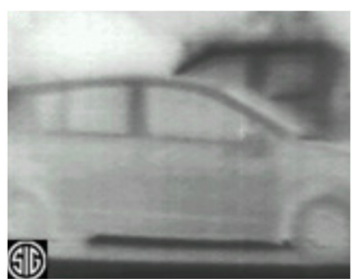

(c)

Fig. 12 (a) GRIN lens was integrated in a thermal reflex sight replacing the original two-element objective with a single GRIN element. The imaging performance of (b) the OE lens surpassed that of (c) the GRIN lens, but the latter was not AR-coated and had a stacking fault degrading its performance.

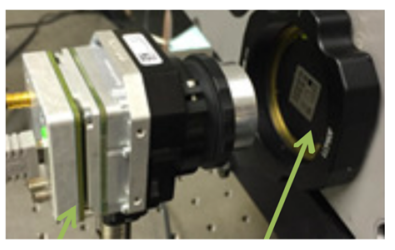

(a)

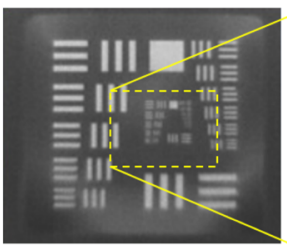

(b)

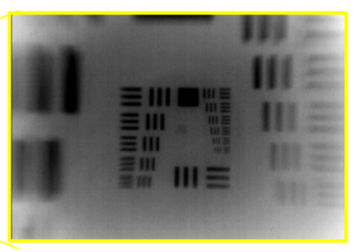

Fig. 13 FLIR Tau2 test bench with (a) GRIN lens objective and (b) resulting image of USAF test pattern bar mask.

The demonstration here represents the first realization of diffusion-based IR-GRIN optics. The imaging tests show that chalcogenide glass optics with internal refractive index gradients can take the place of multiple element lenses in a commercial imaging system, albeit with degraded performance due to a nonoptimized design and lack of AR-coating.

\section{IR-GRIN Design Study}

To date, design exercises with these materials have suggested that the primary virtue of IR-GRIN elements in imager design is the ability to partially correct for chromatic aberration. ${ }^{39}$ To demonstrate, the design and performance of $25-\mathrm{mm}$ focal length, $f / 1.0$ homogeneous, and GRIN singlet lenses for LWIR ( 8 to $12 \mu \mathrm{m}$ ) are considered here. An IR-GRIN lens with an axial GRIN profile and aspheric surfaces is shown in Fig. 14(a). The axial GRIN profile is approximately parabolic and has a $\Delta n$ of 0.3 over its 6.5-mm thickness as shown in Fig. 14(b). A baseline homogeneous NRL-6 glass singlet, $n=3.15227 @ 10.6 \mu \mathrm{m}, V_{8-10 \mu \mathrm{m}}=257$, with aspheric surfaces is used for comparison. Figure 15 shows the spot diagrams for the homogeneous glass

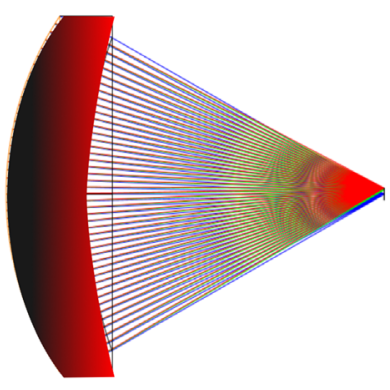

(a)

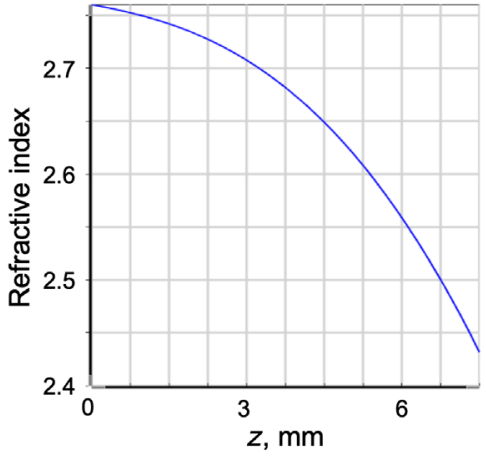

(b)

Fig. 14 (a) LWIR GRIN singlet having (b) a parabolic axial index gradient profile with a $\Delta n=0.3$. 


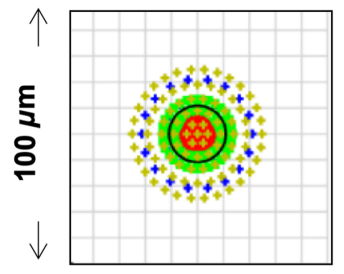

(a)

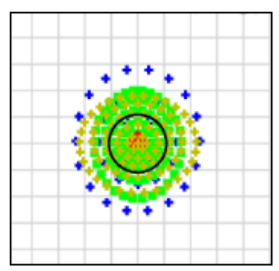

(b)

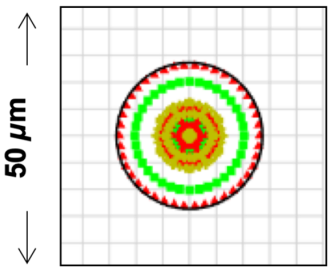

(c)

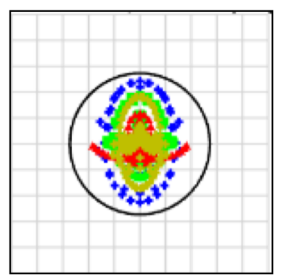

(d)

Fig. 15 Spot diagrams for a homogeneous NRL-6 glass singlet, (a) on-axis and (b) 1-deg off-axis and, at a different scale, spot diagrams for an IR-GRIN singlet, (c) on-axis and (d) 1-deg off-axis. Colored points represent different wavelengths: blue $=8.0 \mu \mathrm{m}$, green $=8.9 \mu \mathrm{m}$, red $=10.4 \mu \mathrm{m}$, yellow $=12.0 \mu \mathrm{m}$. Solid black circle represents the Airy disk at $22 \mu \mathrm{m}$ in diameter.

lens (a) and (b) and the IR-GRIN lens (c) and (d). Note that the diagrams are shown at different scales. In each diagram, the Airy disk is represented by the black circle, and different colored points represent different wavelengths with the LWIR waveband. In the on-axis and 1-deg off-axis diagrams for the homogeneous singlet, Figs. 15(a) and 15(b), respectively, there is significant chromatic aberration and only the red points lie entirely within the Airy disk indicating diffraction-limited performance only near the center of the waveband. The on-axis and 1-deg off-axis diagrams for the IR-GRIN singlet, Figs. 15(c) and 15(d), respectively, reveal greatly reduced chromatic aberration as all points lie within the Airy disk. This lens is diffraction limited across the entire waveband.

In an attempt to evaluate the utility of new homogeneous chalcogenide glasses in singlets, bonded doublets, and IR-GRIN lenses in practical imaging systems, design studies were performed using a variety of notional imager forms including a compact single-band LWIR imager, a cryogenically cooled simultaneous dual-band MWIR/LWIR imager, an uncooled, filterseparable MWIR-LWIR imager, ${ }^{40}$ and a wideband SWIR-LWIR imager. In each example, a baseline design having at most four elements was attempted using commercially available materials and conventional manufacturing methods to fit each notional use case. An alternative design was then attempted utilizing NRL homogeneous glass singlets, bonded elements, and IR-GRIN in combination with the conventional materials, with a design goal of (1) reducing the physical lens element count, (2) reducing the size, weight, and complexity of the lens, and (3) improving the performance or utility of the lens.

\subsection{Single-Band Compact LWIR}

The single-band LWIR design form represents a compact, low-cost imaging application with a $14-\mathrm{mm}$ focal length and $f / 0.85$ lens imaging over 8.5 to $11 \mu \mathrm{m}$. The overall length is constrained to under $15 \mathrm{~mm}$. Three designs are shown in Fig. 16. The baseline design (a) consists of two IRG-26 glass lens elements with aspheric surfaces, fabricated by PGM, and low-cost was prioritized at the expense of degraded performance (d). An improved design (b) with the same configuration achieves diffraction-limited performance (e) through the addition of a diffractive optical element surface on the rear of the front element. In the GRIN design (c), the rear element is replaced with a hybrid-spherical IR-GRIN element $(\Delta n=0.17)$, and the diffractive surface was eliminated from the front element. In this use case, the incorporation of an IR-GRIN element enables diffraction-limited performance $(\mathrm{g})$ without the transmission penalty associated with the diffractive surface and reduced element weight by about $10 \%$.

\subsection{Simultaneous Dual-Band MWIR/LWIR Cryogenically Cooled FPA}

A notional dual-band application utilizing a cryogenically cooled FPA with $1280 \times 720$ pixel format, $12 \mu \mathrm{m}$ pixels, 17.6-mm diagonal, simultaneous imaging in the MWIR and LWIR wavebands, 3.5 to $5.0 \mu \mathrm{m}$, and 7.8 to $10.5 \mu \mathrm{m}$, respectively, is considered. The optical train also incorporates a cold shield as an aperture stop inside the Dewar. The design baseline is a fixed 50-mm focal length, $f / 4$ lens, and the $f / \#$ was decreased incrementally to $f / 1.5 \mathrm{in}$ an attempt to assess the impact of these new materials at lower $f / \#$ where the design is less forgiving. 


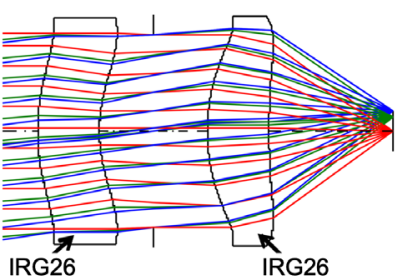

(a)

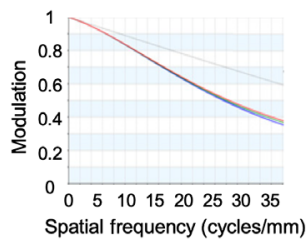

(d)

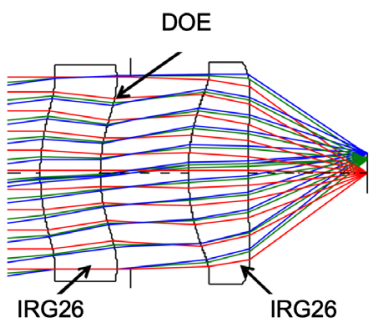

(b)

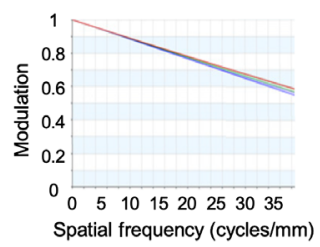

(e)

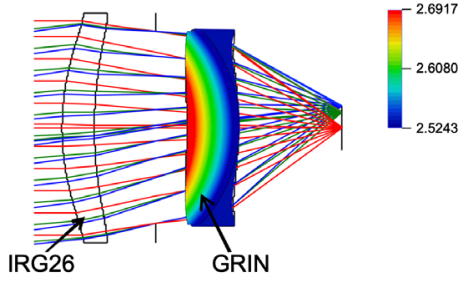

(c)

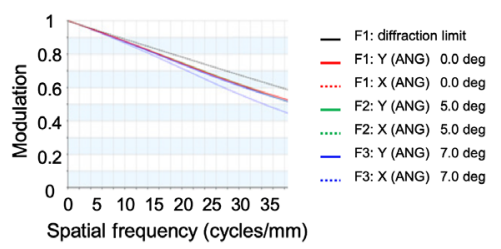

(f)

Fig. 16 Compact LWIR imager designs (a) baseline, (b) diffractive design, and (c) GRIN design and $(\mathrm{e})-(\mathrm{g})$ their respective MTFs.

The baseline $f / 4.0$ lens was designed using only conventional materials and contains three crystalline elements (from front to rear): $\mathrm{ZnSe}$, germanium, and $\mathrm{BaF}_{2}$, and its MTF was nearly diffraction-limited in both the LWIR and MWIR. Redesigning this lens with the new materials, specifically a doublet front element comprising NRL-8 and NRL-6 and a ZnS rear element, yielded a diffraction-limited lens with two fewer surfaces requiring AR coating. There are potential cost savings to be realized by eliminating germanium in favor of glass and reducing the AR-coating operations. In this design, the rear element could be eliminated, leaving only the NRL-8/NRL-6 bonded doublet if the system requirement can tolerate a slightly degraded MTF in the MWIR for the benefit of a much simpler lens design with about $50 \%$ weight savings and only two surfaces to be AR-coated for further reductions in SWaP-C.

At $f / 3.0$, the crystal design required four elements (from front to rear): $\mathrm{ZnSe}$, germanium, $\mathrm{BaF}_{2}$, and germanium to achieve a diffraction-limited MTF. An equivalent performing lens was achieved using a bonded-doublet (NRL-8/NRL-6) front element and a $\mathrm{ZnS}$ rear element. A true two-element solution was found with NRL-4 front element and an IR-GRIN $(\Delta n=0.04)$ rear element, but the MTF was degraded in the MWIR. As the aperture of the design is increased (lower $f / \#$ ), the benefits of the new materials and especially IR-GRIN become more significant. At $f / 1.5$, the MTF performance of the four-element baseline design, Fig. 17(a), is degraded the LWIR, Fig. 17(b), and MWIR wavebands, Fig. 17(c). Utilizing the new materials, a 50-mm, $f / 1.5$ lens with three physical elements (from front to rear): NRL-8/NRL-6 bonded doublet, a $\mathrm{ZnS}$ singlet, and an IR-GRIN rear element with a $\Delta n=0.12$, was designed. The lens design is shown in Fig. 18(a). The radial GRIN profile for the IR-GRIN element is shown in Fig. 18(b) where each color represents the GRIN profile at different wavelengths as indicated in the legend. In this doublet + singlet + GRIN design, the MTF performance was recovered in the LWIR and

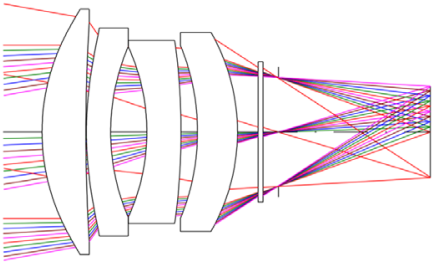

(a)

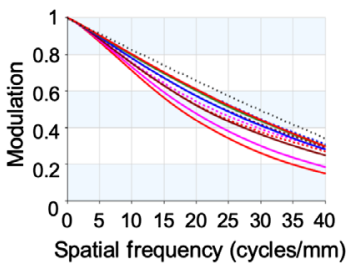

(b)

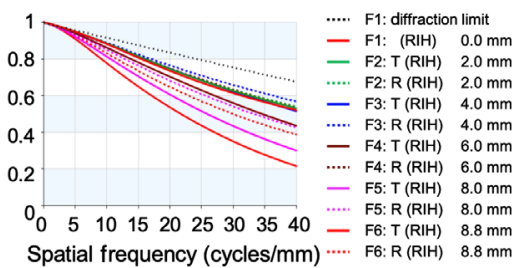

(c)

Fig. 17 (a) Baseline dual-band MWIR-LWIR 50-mm, f/1.5 lens design having four conventional elements, from front to rear, $\mathrm{ZnSe}$, germanium, $\mathrm{BaF}_{2}$, and germanium. The MTF of this design was calculated for (b) the LWIR and (c) MWIR wavebands. 


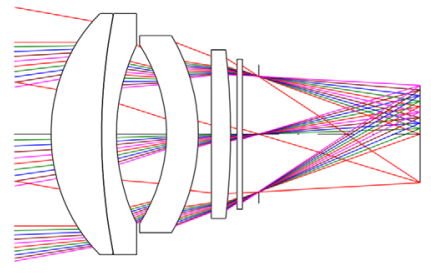

(a)

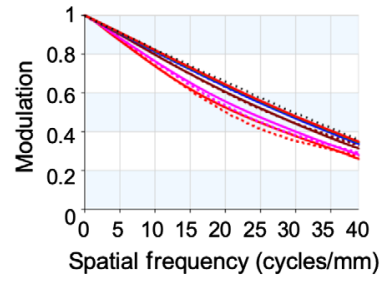

(c)

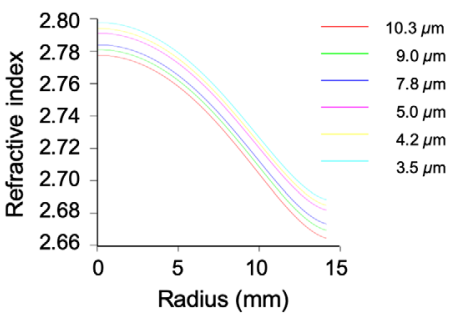

(b)

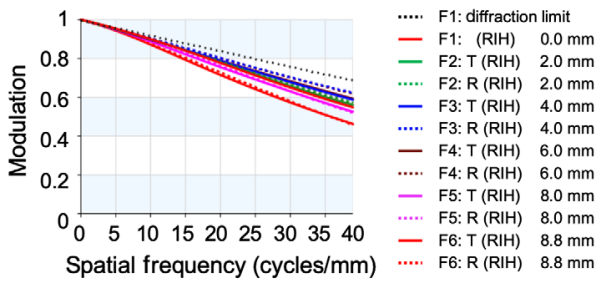

(d)

Fig. 18 (a) Redesigned 50-mm, $f / 1.5$ lens design utilizing the new NRL materials, from front to rear, bonded NRL-8/NRL-6 doublet, ZnS, and IR-GRIN with a radial profile (b). The calculated MTFs in (c) the LWIR and (d) MWIR are improved over the baseline design and approach the diffraction limit (dotted black line).

MWIR wavebands as shown in Figs. 18(c) and 18(d), respectively, and is nearly diffraction limited in both wavebands. The doublet + singlet + GRIN lens is $18 \%$ shorter, $15 \%$ lighter in weight, and has two fewer surfaces for AR coating. A third lens was designed exclusively using IR-GRIN elements as shown in Fig. 19(a). This all-GRIN design employed front and rear IR-GRIN elements with different complementary radial GRIN profiles as shown in Figs. 19(b) and 19(c), respectively. The GRIN profiles are complementary in that the front element $(\Delta n=0.06)$ has its low refractive index at the center and the rear element $(\Delta n=0.25)$ has its high refractive index at the center. The all-GRIN lens is similar in size and weight to the doublet + singlet +GRIN design. The MTF performance of the all-GRIN lens design exceeds that of the baseline design, in both the LWIR and MWIR wavebands as shown in Figs. 19(d) and 19(e),

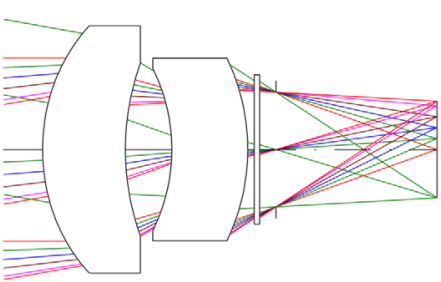

(a)

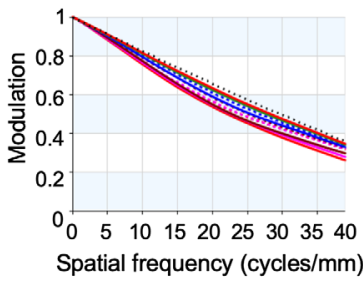

(d)

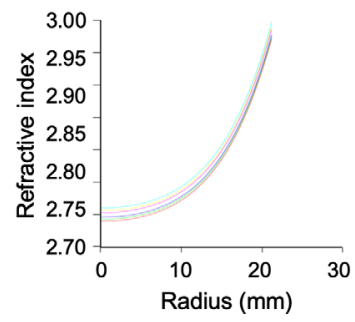

(b)

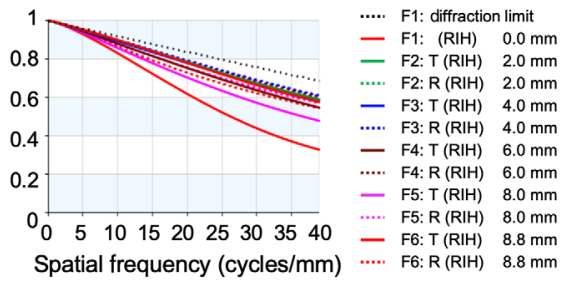

(e)

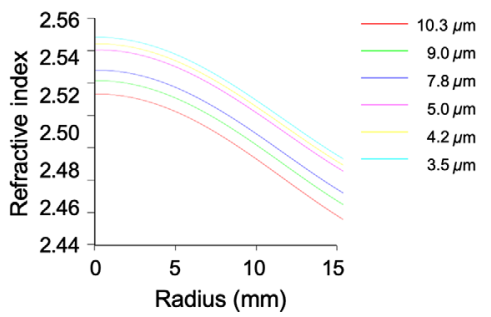

(c)

Fig. 19 (a) An advanced 50-mm, $f / 1.5$ lens design utilizes only two lens elements: (b) a front IR-GRIN with a radial profile and (c) a rear IR-GRIN with a complementary radial profile. (d) The calculated MTF is nearly diffraction limited in the LWIR waveband and has good performance in (e) the MWIR waveband for most of the field, excluding the periphery. 
respectively, but is slightly degraded when compared to the doublet + singlet + GRIN design of Fig. 18.

This design exercise highlights some of the advantages and limitations of designing with these new IR glasses in GRIN and doublet form. While the all-GRIN design contains fewer lens elements, which reduces the number of surfaces needing AR-coatings and allows for a much-simplified assembly, the MTF performance, while better than the baseline design, is slightly degraded compared to the doublet + singlet + GRIN design. The use of the NRL glasses enables bonded doublets and IR-GRIN to be used alongside conventional materials for smaller, lighter optics with better performance and fewer surfaces requiring AR-coatings, especially at low $f / \#$.

\subsection{Filter-Separable Dual-Band MWIR-LWIR Uncooled FPA}

Another application utilizing a notional dual-band uncooled FPA was considered. Arrays of this type are broadband ( 3.5 to $12 \mu \mathrm{m}$ ), and the MWIR and LWIR bands are only separable using filters. The notional FPA considered here is $640 \times 480$ pixels with a $12-\mu \mathrm{m}$ pixel size and a 10-mm diagonal. As the FPA is uncooled and lacks a cold shield, the aperture stop may be located anywhere in the lens. As the small pixel size necessitates a fast $f /$ number lens, $f / 1.4-f / 1.0$ were investigated for the 50 -mm focal length lens.

Since the FPA in this example is capable of LWIR capture out to $12 \mu \mathrm{m}$, materials such as $\mathrm{CaF}_{2}$ and $\mathrm{BaF}_{2}$ were eliminated from use. The baseline 50-mm, $f / 1.4$ lens contained four crystalline elements (from front to rear): $\mathrm{ZnSe}, \mathrm{GaAs}, \mathrm{ZnS}$, and GaAs as shown in Fig. 20(a). The combined MWIR-LWIR MTF was calculated for the baseline design, shown in Fig. 20(b), and indicates a poor performing lens, although the performance is consistent across the field. In this design exercise, lens designs utilizing the NRL materials were constrained to two physical lens elements and an overall length shorter than that of the baseline lens in an attempt to provide significant size and weight reductions. A 50-mm, $f / 1.4$ lens utilizing the NRL materials was designed having an NRL-8/NRL-6 doublet front element and a ZnS rear element and is shown in Fig. 21(a). This lens was much more compact, $27 \%$ shorter in overall length, and $40 \%$ lighter in weight compared to the baseline lens and had much better performance as the combined MTF,

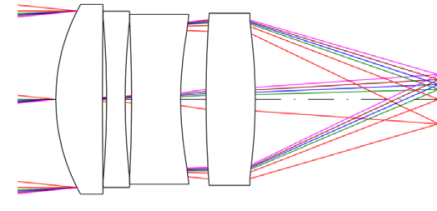

(a)

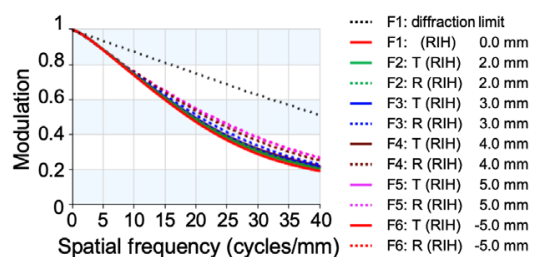

(b)

Fig. 20 (a) Baseline, all-crystalline, 50-mm $f / 1.4$ lens designed for a notional uncooled dual-band MWIR/LWIR detector with four elements: ZnSe, GaAs, ZnS, and GaAs (front to rear). (b) The calculated MTF for this lens at the full 3.5- to $12-\mu \mathrm{m}$ combined spectrum.

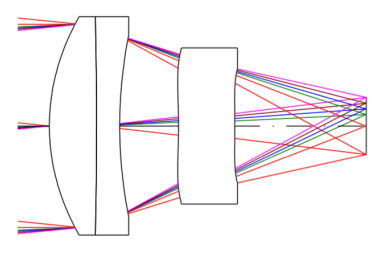

(a)

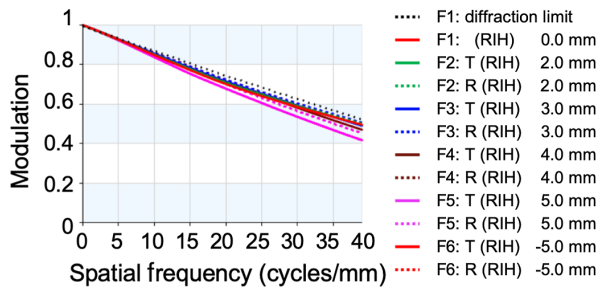

(b)

Fig. 21 (a) A 50-mm $f / 1.4$ lens using the NRL materials in a doublet + singlet configuration has a bonded NRL-8/NRL-6 doublet and a ZnS rear element (front to rear). (b) The combined MTF for this design is nearly diffraction limited across the full 3.5 to $12 \mu \mathrm{m}$ spectrum. 


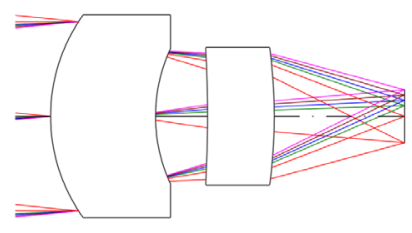

(a)

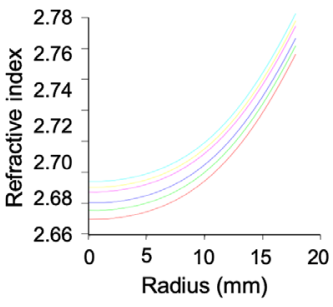

(c)

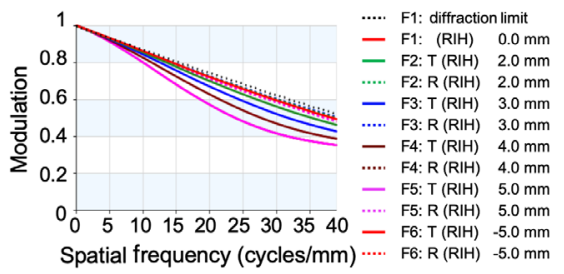

(b)

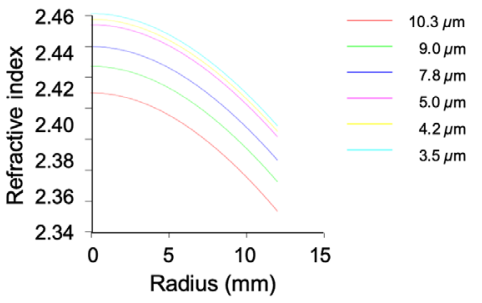

(d)

Fig. 22 (a) A 50-mm $f / 1.4$ lens in an all-GRIN configuration utilizes a front GRIN with $\Delta n=0.06$ and a complementary rear GRIN with $\Delta n=0.09$. (b) The combined MTF is nearly diffraction limited in the center of the field, with some distortion at the periphery. The radial GRIN profiles of (c) the front and (d) rear elements are complementary.

Fig. 21(b), was approximately diffraction-limited across the field. Figure 22(a) shows the design of an all-GRIN lens with two different front and rear IR-GRIN elements. The radial GRIN profiles of the two elements in this design, (c) and (d), respectively, are complementary in that the front element $(\Delta n=0.06)$ has its low index at the center and the rear elements $(\Delta n=0.09)$ have their high index at the center. This all-GRIN lens is $13 \%$ shorter in overall length compared to the baseline. The combined MTF for the all-GRIN $f / 1.4$ lens is shown in Fig. 22(b) and was significantly better than the baseline, but slightly degraded compared to the doublet + singlet lens, notably at the periphery of the field.

The doublet + singlet and all-GRIN lens design forms were used as starting points that were reoptimized for wider aperture lenses at $f / 1.2$ and $f / 1.0$. The baseline all-crystalline lens, due to its extremely poor performance at $f / 1.4$, was not developed further at the more challenging lower $f /$ numbers. At $f / 1.2$, the doublet + singlet MTF performance, shown in Fig. 23(a),

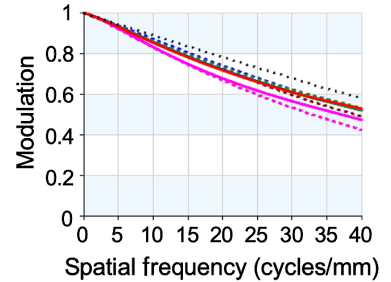

(a)

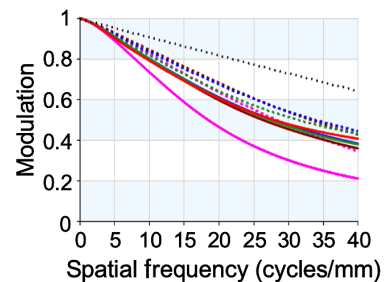

(d)

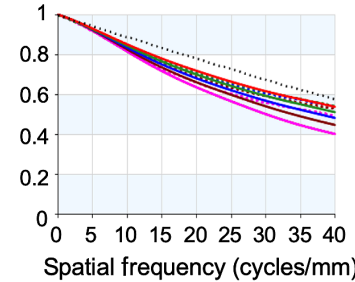

(b)

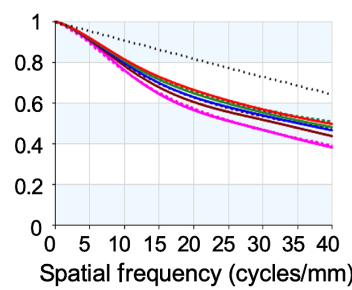

(e)

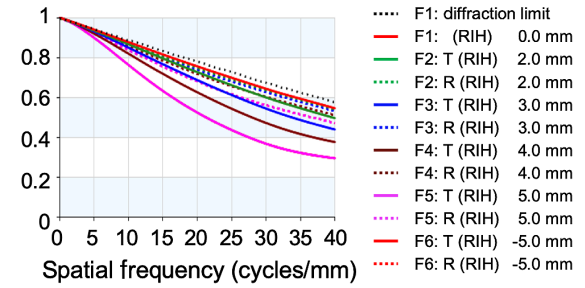

(c)

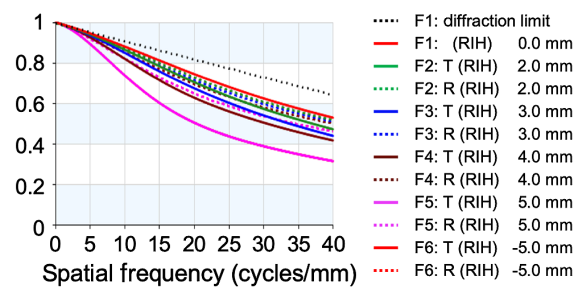

(f)

Fig. 23 Combined MTFs calculated for 50-mm dual-band lenses designed for an uncooled 3.5 to $12 \mu \mathrm{m}$ detector in six different configurations: (a) $f / 1.2$ doublet + singlet, (b) $f / 1.2$ doublet + GRIN, (c) $f / 1.2$ all-GRIN, (d) $f / 1.0$ doublet + singlet, (e) $f / 1.0$ doublet + GRIN, and (f) $f / 1.0$ all-GRIN. 


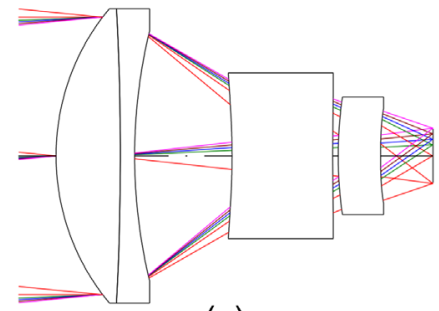

(a)

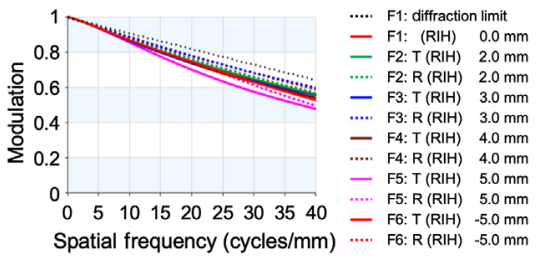

(b)

Fig. 24 A 50-mm $f / 1.0$ dual-band lens in a doublet +2 singlets configuration (a) based on the doublet + singlet design shown in Fig. 21(a) and has three physical elements: a bonded NRL-8/ NRL-6 doublet, a ZnS singlet, and an NRL-6 singlet (from front to rear). (b) The combined MTF of this lens is approximately diffraction limited.

was similar to the $f / 1.4$ version, Fig. 21(b), but slightly degraded. An attempt was made to restore performance by replacing the $\mathrm{ZnS}$ singlet with an IR-GRIN element $(\Delta n=0.06)$ to make a doublet + GRIN design. The combined MTF, Fig. 23(b), did not change very much; it was slightly improved at the center of the field and some astigmatism was introduced. The $f / 1.2$ all-GRIN design, with a front element $\Delta n=0.06$ and a rear element $\Delta n=0.14$, had a combined MTF, Fig. 23(c), similar to its $f / 1.4$ counterpart, Fig. 22, but with some astigmatism and degradation at the periphery of the field. Opening the aperture to $f / 1.0$ begins to reveal the limitations of these design forms as shown in Figs. 23(d)-23(f), largely due to the low lens element count design constraint. The doublet + singlet lens design suffered the largest degradation in its combined MTF, Fig. 23(d). The MTF of the $f / 1.0$ doublet + GRIN $(\Delta n=0.04)$ design, shown in Fig. 23(e), is similar to the $f / 1.0$ doublet + singlet design, but with reduced astigmatism and blur at the periphery. The MTF of the $f / 1.0$ all-GRIN lens design as shown in Fig. 23(f) is only slightly degraded compared to its $f / 1.2$ counterpart, most likely due to the more severe index gradient of the rear element $(\Delta n=0.25)$, but is only a slight improvement over the $f / 1.0$ doublet + GRIN design. It should be noted that even though the combined MTF of these designs at $f / 1.0$ is not quite diffraction-limited, all designs outperform the baseline design at $f / 1.4$. This implies a significant potential for improved lens performance in a reduced footprint compared to conventional materials in more demanding applications.

In an attempt to recover the performance lost at $f / 1.0$, the lens element count constraint was relaxed and an additional singlet was added to the doublet + singlet design form of Fig. 23(d). The resulting doublet +2 singlet design is shown in Fig. 24(a) and contains (from front to rear): an NRL-8/NRL-6 bonded doublet, a ZnS singlet, and an NRL-6 singlet. The combined MTF of this lens is shown in Fig. 24(b) and approaches the diffraction limit.

This design exercise shows that for dual-band applications requiring low $f /$ numbers, the incorporation of these new materials alongside conventional materials can result in compact lenses with combined MTF performance near the diffraction limit. Increasing the $\Delta n$ of GRIN elements can be used to improve or maintain performance at low- $f$ /\# but can introduce some astigmatism. One unexpected result was that the doublet +2 singlet design of Fig. 24 at $f / 1.0$ significantly outperformed the baseline all-crystal design $f / 1.4$ lens with an equivalent number of refractive surfaces. Using these materials allows for more compact, lower- $f$ /\# dual-band optics than are possible with conventional materials. In addition, GRIN and bonded doublets allow for simpler lens assemblies since the optical complexity has been offloaded to the lens element construction.

\subsection{Broadband XSWIR/MWIR/LWIR}

Broadband lens designs, for example, those tasked with operation over SWIR, MWIR, and LWIR wavebands, can be become very complex with many elements. ${ }^{1}$ As the target wavelength range becomes large, chromatic aberration becomes the limiting obstacle. This is compounded further by the small number of broadband compatible refractive materials. In this challenging design case, a notional $640 \times 512$ uncooled FPA with $12-\mu \mathrm{m}$ pixels and a $10-\mathrm{mm}$ diagonal, operating at 1.5 to $12 \mu \mathrm{m}$ was considered. The lens design requirement was a $50-\mathrm{mm}$ focal length, 


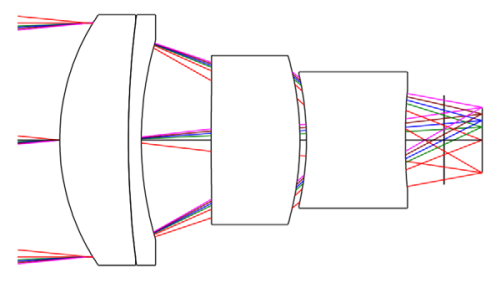

(a)

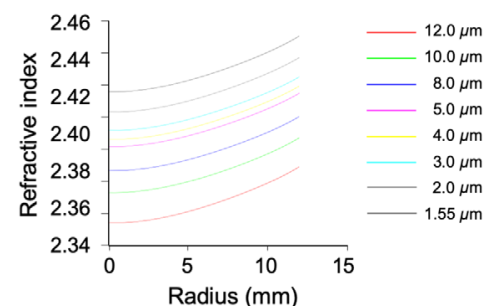

(b)

Fig. 25 (a) Broadband lens design with a 50-mm focal length, $f / 1.2$, and comprises four refractive elements including (from left) bonded NRL-8/NRL-6 doublet, IR-GRIN element, and ZnS rear element. The radial profile of the IR-GRIN element is shown in (b).

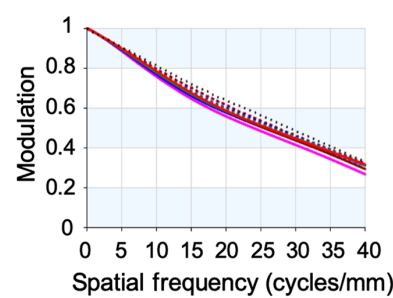

(a)

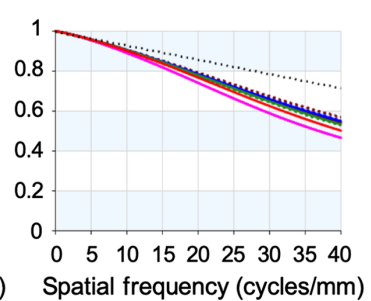

(b)

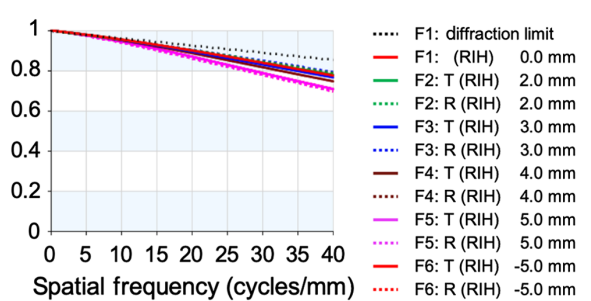

(c)

Fig. 26 MTFs for the broadband lens optimized for separable FPAs at (a) LWIR, (b) MWIR, and (c) XSWIR wavebands.

$f / 1.2$. A baseline design using only conventional refractive materials was not achieved. The lens design using NRL materials, shown in Fig. 25(a), contains three physical elements (from front to rear): an NRL-8/NRL-6 bonded doublet, an IR-GRIN element, and a $\mathrm{ZnS}$ rear element. The IR-GRIN element has a mild radial profile, Fig. 25(b), with $\Delta n=0.03$. In this design concept, the surfaces were optimized for separable FPAs resulting in an MTF performance that is approximately diffraction limited in LWIR and extended short-wavelength infrared (XSWIR) wavebands, Figs. 26(a) and 26(c), respectively, and slightly degraded in the MWIR waveband as in Fig. 26(b). This design form was also optimized for a filter-separable common multiband FPA, which degrades the combined broadband MTF.

\section{Conclusions}

The pursuit of diffusion-based IR-GRIN glass optics has brought about a multitude of benefits for SWIR-LWIR imaging. A series of moldable chalcogenide glasses for SWIR-LWIR imaging has been developed and expanded the glass map for dual-band and wideband IR imager designs, as shown in Fig. 27. These glass maps show the refractive optical materials compatible with dualband MWIR-LWIR (a), and wideband SWIR-LWIR lens design (b), plotted as the average refractive index over the band of interest (ordinate) against the average dispersion over the same band (abscissa). The plots include commonly available crystals (orange squares), common glasses (blue diamonds), and the glasses developed in this work (green circles) with the IRGRIN compatible glasses outlined in black. In addition to being moldable, these glasses have similar glass transition temperatures and similar thermal expansion coefficients enabling thermal bonding of lens elements to create bonded doublets, triplets, and multilayered lenses without the need for IR-compatible adhesives. These glasses were used in the design, fabrication, and demonstration of a dual-band MWIR-LWIR imager, ${ }^{41}$ and the lens element count was reduced by $50 \%$ due to the use of these materials in singlet and bonded doublet forms. The costs are further reduced due to fewer surfaces requiring AR coating and reduced assembly tolerances arising from the smaller element count.

IR-GRIN optics with continuous index gradients were fabricated for the first time in axial and hybrid-spherical configurations and demonstrated in a commercial imaging system. 


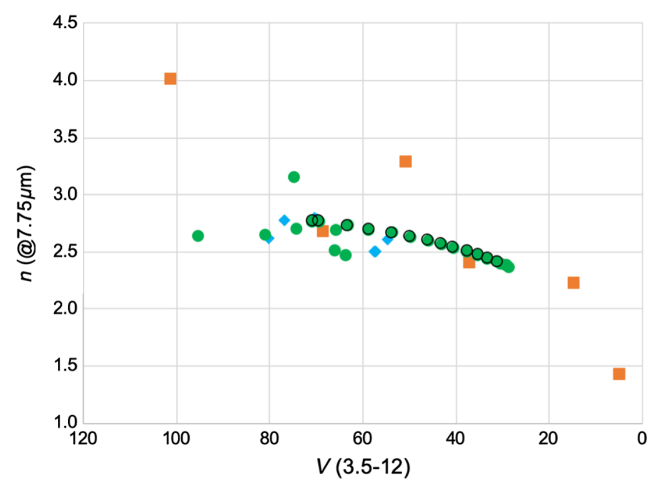

(a)

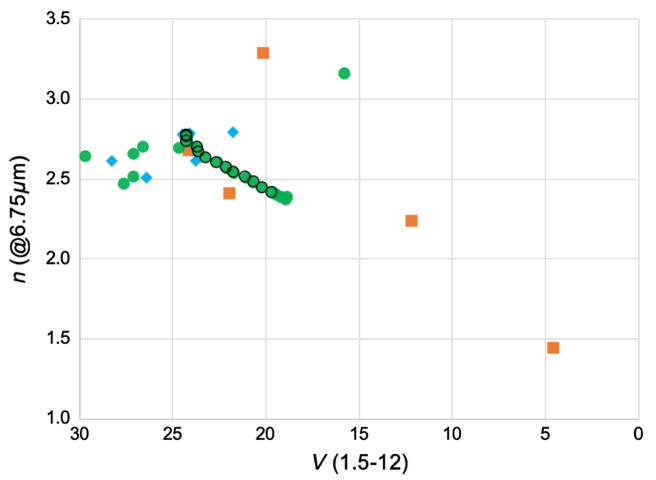

(b)

Fig. 27 Glass maps showing the commonly available crystals (orange squares) and glasses (blue diamonds) compatible with (a) dual-band MWIR-LWIR (3.5 to $12 \mu \mathrm{m}$ ) and (b) wideband SWIRLWIR (1.5 to $12 \mu \mathrm{m}$ ) lens design. Glasses developed in this work expand the maps and are shown as green circles (GRIN-compatible glasses are outlined in black). Materials that are especially toxic or hygroscopic (e.g., $\mathrm{TIBr} 42158, \mathrm{KBr}, \mathrm{NaCl}$ ) are omitted.

Fabrication of IR-GRIN lens elements with purely radial GRIN profiles is planned for future work. The IR-GRIN lens demonstrated here had a $\Delta n$ of 0.2 , and the system is capable of a $\Delta n$ of 0.4. The principle advantages of using IR-GRIN in optics design arise from the difficulty in color correction across wide wavelength ranges using conventional optics. To that end, the most substantial SWaP-C benefit potential lies in the dual-band and wideband IR applications and more demanding configurations including wide FOV and zoom systems.

Lens design exercises demonstrate the potential for improving the performance of compact lenses for dual-band and wideband imaging applications compared to conventional materials. New homogeneous glasses, bonded doublets, and IR-GRIN (with axial, hybrid-spherical, and radial GRIN profiles) can reduce the lens element count and enable lower $f$ /\# lenses in the same or smaller footprints compared with conventional designs. With the improved performance and reduced complexity come the potential for reduced cost as high-volume PGM processes can replace costly single point diamond turning of crystalline elements.

\section{Acknowledgments}

The authors would like to thank Jamie Ramsey, George Lindberg, John Deegan, and Blair Unger, at RPO for their collaborative efforts fabricating optics, modeling, design, test, and demo. Dean Scribner and Val Maksimovic at Northrop Grumman collaborated on design and test of the laminated optic. Jeremey Teichman at the Institute for Defense Analysis (Alexandria, Virginia) provided insight regarding diffusion and optical modeling. The views, opinions, and/or findings expressed are those of the author(s) and should not be interpreted as representing the official views or policies of the Department of Defense or the U.S. Government. Distribution Statement A, Approved for Public Release, Distribution Unlimited.

\section{References}

1. S. Sparrold et al., "Refractive lens design for simultaneous SWIR and LWIR imaging," Proc. SPIE 8012, 801224 (2011).

2. S. D. Gunapala et al., " $1024 \times 1024$ pixel mid-wavelength and long-wavelength infrared QWIP focal plane arrays for imaging applications," Semicond. Sci. Technol. 20(5), 473480 (2005).

3. D. J. Gibson, S. S. Bayya, and J. S. Sanghera, "Homogeneous and gradient index (GRIN) materials for multi-band IR optics," in Classical Opt., paper IW2A.1, Optical Society of America, Washington, D.C. (2014).

4. S. S. Bayya et al., "Design and fabrication of multispectral optics using expanded glass map," Proc. SPIE 9451, 94511N (2015). 
5. D. J. Gibson et al., "IR-GRIN optics for imaging," Proc. SPIE 9822, 98220R (2016).

6. G. Beadie, E. Stover, and D. J. Gibson, "Temperature-dependent dispersion fitting for a recent infrared glass catalog," Proc. SPIE 10998, 1099804 (2019).

7. J. A. Corsetti, P. McCarthy, and D. T. Moore, "Color correction in the infrared using gradient-index materials," Opt. Eng. 52(11), 112109 (2013).

8. R. C. Augusteyn and A. Stevens, "Macromolecular structure of the eye lens," Prog. Polym. Sci. 23(3), 375-413 (1998).

9. D. E. Nilsson et al., "Advanced optics in a jellyfish eye," Nature 435, 201-205 (2005).

10. L. Garner et al., "Gradient refractive index of the crystalline lens of the Black Oreo Dory (Allocyttus niger): comparison of magnetic resonance imaging (MRI) and laser ray-trace methods," Vision Res. 41(8), 973-979 (2001).

11. J. A. Corsetti et al., "Design, fabrication, and metrology of polymer gradient-index lenses for high-performance eyepieces," Proc. SPIE 8841, 88411G (2013).

12. A. J. Visconti, D. T. Moore, and J. L. Bentley, "Large diameter radial gradient-index lenses fabricated by ion exchange," in Imaging and Applied Optics Technical Papers (2012).

13. P. McCarthy, N. Nag, and D. T. Moore, "Modeling mid-spatial frequency wavefront error in gradient-index ALON fabricated by layered diffusion," in Classical Opt., paper IW2A.4, Optical Society of America, Washington, D.C. (2014).

14. B. V. Hunter et al., "Current developments in GRADIUM® glass technology," Proc. SPIE 3482, 789-800 (1998).

15. J. R. Hensler, "Method of producing a refractive index gradient in glass," 3,873,408 (1975).

16. S. Ji et al., "A bio-inspired polymeric gradient refractive index (GRIN) human eye lens," Opt. Express 20(24), 26746-26754 (2012).

17. J. Teichman et al., "Gradient index optics at DARPA," D-5027, pp. 1-69 (2013).

18. D. J. Gibson et al., "GRIN optics for multispectral infrared imaging," Proc. SPIE 9451, 94511P (2015).

19. L. Sisken et al., "Examination of laser-induced heating on multi-component chalcogenide glass," Proc. SPIE 9070, 90701A (2014).

20. D. H. Werner et al., "Adaptive phase change metamaterials for infrared aperture control," Proc. SPIE 8165, 81651H (2011).

21. K. Richardson et al., "Advances in infrared GRIN: a review of novel materials towards components and devices," Proc. SPIE 10627, 106270A (2018).

22. L. Sisken et al., "Evidence of spatially selective refractive index modification in $15 \mathrm{GeSe}_{2}-45 \mathrm{As}_{2} \mathrm{Se}_{3}-40 \mathrm{PbSe}$ glass ceramic through correlation of structure and optical property measurements for GRIN applications," Opt. Mater. Express 7, 3077-3092 (2017).

23. L. Sisken et al., "Infrared glass-ceramics with multidispersion and gradient refractive index attributes," Adv. Funct. Mater. 29, 1902217 (2019).

24. E. Lavanant et al., "Radial gradient refractive index (GRIN) infrared lens based on spatially resolved crystallization of chalcogenide glass," Opt. Mater. Express 10, 860-867 (2020).

25. S. Novak et al., "Direct electrospray printing of gradient refractive index chalcogenide glass films," ACS Appl. Mater. Interfaces 9(32), 26990-26995 (2017).

26. S. D. Campbell et al., "Three-dimensional gradient-index optics via injket aided additive manufacturing techniques," in IEEE Int. Symp. Antennas and Propagation and USNC/URSI Natl. Radio Sci. Meeting, IEEE, pp. 605-606 (2015).

27. A. Lepicard et al., "Long-lived monolithic micro-optics for multispectral GRIN applications," Sci. Rep. 8, 7388 (2018).

28. J. S. Sanghera et al., "Layered chalcogenide glass for IR optics," in MSS Passive Sens., Pasadena (2012).

29. D. S. Cary, W. F. Parsons, and E. Carnall, "Infrared lens cement," 3,157,521, U.S. Patent, pp. 1-2 (1964).

30. S. F. Pellicori, "Optical bonding agents for IR and UV refracting elements," Proc. SPIE 1535, 48 (1991).

31. S. M. Song et al., "Chalcogenide glasses for optical brazing," J. Mater. Sci. 33, 5397-5400 (1998).

32. K. S. Prabhu et al., "A survey of technical literature on adhesive applications for optics," Proc. SPIE 6665, 666507 (2007). 
33. N. Carlie, "Optical bonding through the use of low-softening point optical glass for IR optical glass for IR optical applications and products formed," 10,191,186, U.S. Patent, 2nd ed., pp. 1-8 (2019).

34. D. J. Gibson et al., "Layered chalcogenide glass structures for IR lenses," Proc. SPIE 9070, 90702I (2014).

35. J. L. Ramsey, B. Unger, and G. Lindberg, "Design study of a MWIR/LWIR multiple FOV lens," Proc. SPIE 10627, 1062703 (2018).

36. G. P. Lindberg et al., "Raman and CT scan mapping of chalcogenide glass diffusion generated gradient index profiles," Proc. SPIE 9822, 98220W (2016).

37. G. P. Lindberg et al., "Methods of both destructive and non-destructive metrology of GRIN optical elements," Proc. SPIE 9451, 94511 S (2015).

38. N. Y. Gnedin, V. A. Semenov, and A. V. Kravtsov, "Enforcing the Courant-Friedrichs-Lewy condition in explicitly conservative local time stepping schemes," J. Comput. Phys. 359, 93-105 (2018).

39. D. J. Gibson et al., "IR GRIN optics: design and fabrication," Proc. SPIE 10181, 101810B (2017).

40. D. J. Gibson et al., "Multispectral IR optics and GRIN," Proc. SPIE 10998, 109980D (2019).

41. J. Ramsey et al., "Comparison study of a multispectral zoom lens using standard and novel optical materials," Appl. Opt. 58(18), 5045-5046 (2019).

Daniel Gibson is a research scientist in the Optical Materials and Devices Branch at the U.S. Naval Research Laboratory in Washington, DC, USA, where he has been developing infrared optical materials and fibers since 2003. He received his $\mathrm{PhD}$ in ceramic and materials engineering from Rutgers University in 2003. His research interests include infrared materials, fiber optics, devices, lasers, and sensors. He is the author of over 30 journal articles, has written one book chapter, and holds over a dozen patents, several of which are licensed to industry.

Shyam Bayya received his PhD in ceramics from Alfred University, Alfred, NY, in 1991. He joined NRL in 1994 and has worked on optical glasses and ceramics for various DOD applications. His research interests are in infrared glasses including silicates, germanates, tellurides and chalcogenides, active and passive IR fiber optics and transparent ceramics for IR domes and sensor windows. He has published more than 80 papers and has 39 awarded patents.

Jason Myers is a materials research engineer at the Naval Research Laboratory. He currently manages the NRL Advanced Thin Films Laboratory and maintains a research portfolio including novel optical materials development, non-mechanical beam steering devices, thin film materials science, optoelectronics, and metamaterials.

Jas Sanghera is branch head of optical materials and devices at the Naval Research Laboratory in Washington, DC, where he is responsible for the development of unique capabilities addressing a wide range of DoD applications. He has published over 340 papers, edited a book on IR fiber optics, presented more than 250 technical talks, and has $>110$ patents awarded and 59 licensed to industry. He is a fellow of both OSA and SPIE.

Guy Beadie is director of optical technologies at Peak Nano Optics, LLC, which is developing new technology in gradient index (GRIN) optics. For 21 years before his current position, he was a Research Scientist in the Optical Sciences Division of the U.S. Naval Research Laboratory. Research interests included nonlinear optics and ultrafast dynamics in organic molecules. For the past 15 years, his primary interests have been in polymer and GRIN optics.

Biographies of the other authors are not available. 